\title{
Pilot to improve the appropriate prescription of oral nutritional supplements within the Walsall area
}

\author{
R. Ragubeer ${ }^{1}$ and H. J. Patel ${ }^{2}$ \\ ${ }^{1}$ Walsall Healthcare NHS Trust, Walsall, WS2 9PS, UK and ${ }^{2}$ Jubilee House, NHS Walsall, Walsall, UK
}

Growth in expenditure of oral nutritional supplements (ONS), which are commonly used to treat malnutrition, has risen substantially in recent years. A high proportion of supplements (57-75\%) are prescribed inappropriately, resulting in significant waste and unnecessary healthcare costs ${ }^{(1)}$. Despite this significant cost, evidence suggests that recognition of malnutrition and monitoring of patients in the community is poor $^{(2)}$ and ONS are often used inappropriately ${ }^{(3)}$. Assessing the appropriateness of ONS expenditure in the community falls into the NHS QIPP (Quality, Innovation, Productivity and Prevention) agenda.

A pilot project was completed at a GP surgery in Walsall to ensure ONS were appropriately prescribed and to reduce the cost associated with inappropriate prescribing. Expected outcomes were as follows:

1) To ensure that patients who were prescribed nutritional supplements had a nutritional assessment

2) To identify patients who no longer required prescribable oral supplements

3) To ensure patients who did require nutritional supplements were prescribed the appropriate type and volume

4) To share the findings with all GP practices, GP consortia and PCT Medicines Management Committee

The lead GP and practice based pharmacist identified patients at the surgery who had been prescribed ONS in the past 12 months using a simple computer search. Search criteria excluded patients who were tube fed and palliative patients. A baseline audit was completed by the clinical community dietitian and practice based pharmacist on the remaining list of patients using the patient information on the GP clinical system (EMIS LV) to locate the original assessments and recommendations/prescriptions for ONS. Results showed that most of the patients had no nutritional assessment documented on initiation of ONS. Those who were found to be palliative or already known to the dietitians were identified and all remaining patients were sent clinic appointment letters and patient questionnaires. For the patients who could not attend the clinic, a home visit was offered.

A questionnaire was also given to all GP's, practice nurses and other healthcare professionals who were involved with the prescribing of ONS. The practice based pharmacist generated data identifying the ONS spend on these patients before and after dietetic assessment.

Of the 25 patients prescribed ONS, 4 patients were palliative and 1 had cancer (none of these patients had been asked to stop their supplements); 3 patients did not attend their appointments; 4 patients were referred to specialist dietitians; 4 patients continued on current regimen; 8 patients were found to be well nourished and had their ONS discontinued and 1 patient had left the surgery. The total annualised cost savings from discontinuing the ONS after dietetic assessment was $£ 20454.60$.

The results from the patient questionnaire revealed that $75 \%$ were initiated by GP's or hospital doctors, $75 \%$ were on supplements for more than 6 months, $50 \%$ of those asked thought the supplements were to gain weight and $63 \%$ claimed to have never had a review regarding their supplements. The results from the health professional questionnaire revealed that $80 \%$ claimed they were aware of the local malnutrition guidelines, $60 \%$ believed the patients were regularly monitored using a screening tool such as MUST, 50\% would prefer to involve a dietitian in the initial prescription of ONS and $75 \%$ noted they would like some form of training on the supplements and how to involve a dietitian within the process.

The pilot shows that without dietitian intervention the 25 patients would have continued on their current ONS without review to assess them clinically and to either make recommendations, alter treatment options, alter quantities of the current ONS or discontinue treatment. An ongoing specialist dietetic service is important to maintain improvements in practice. Dietitians are more effective than GP's at offering support to patients and helping to reduce inappropriate prescription ${ }^{(1)}$.

1. London Procurement Programme Clinical Oral Nutrition Support Project (January 2009).

2. Loane D, Flanagan G, Siun A, McNamara E \& Kenny S (2004) Nutrition in the community - an exploratory study of oral nutritional supplements in a health board area in Ireland. $J$ Hum Nutr Diet 17, 257-266.

3. Kennelly S, Kennedy NP, Flanagan Rughoobur G, Glennon Slattery C \& Sugrue S (2009) The use of oral nutritional supplements in an Irish community setting. J Hum Nutr Diet 22(6):511-520. 